and for adenylate kinase from pig, as opposed to a positive $\Delta \mathrm{pI}$ for the latter enzyme from rat. A small negative $\Delta p I$ of the malate dehydrogenases in yeast contrasts with large positive $\Delta \mathrm{pIs}$ in Neurospora crassa and rabbit. The only significantly negative $\Delta \mathrm{pI}$ is found in the case of rat liver aldehyde dehydrogenase, but this again contrasts with a positive $\Delta \mathrm{pI}$ for the sheep liver enzyme.

Depending on the protein, the observed difference in $\mathrm{pI}$ is due either to more basic amino-acid residues, or to fewer acidic residues or to both in the mitochondrial isoprotein (data available on request). Comparisons of the aminoacid sequences do not reveal any preferential distribution of the positive and negative charges or of the charge differences between the two isoenzymes along the polypeptide chain.

The evolutionary selection for positive charge in mitochondrial isoproteins probably relates either to facilitated importation into this organelle or to adaptation to conditions inside mitochondria.

Biochemisches Institut,

Universität Zürich,

CH-8057 Zürich, Switzerland

ROLF JAUSSI

Paul Scherrer Institut,

CH-5232 Villigen-PSI, Switzerland

1. Verner, K. \& Schatz, G. Science 241, 1307-1312 (1988)

2. Harti, F.-U. \& Neupert, W. Science 247, 930-938 (1990).

3. Hartmann, C., Lindenmann, J.-M., Christen, P. \& Jaussi, R. Biochem. biophys. Res. Commun. 174, 1232-1238 (1991)

4. Malamud, D. \& Drysdale, J. W. Analyt. Biochem. 86 , 620-647 (1978).

5. Righetti, P. G., Tudor, G. \& Ek, K. J. Chromatogr. 220 , 115-194 (1981).

\section{Classical records}

SIR - Ian Percival's suggestion (Nature 351, 357; 1991) that DNA provides an example of a classical measurement embodied in a microscopic system raises some questions. It would appear that any expression of the alteration of a DNA molecule requires a considerable amplification. For example, Percival mentions leukaemia, but leukaemia is not a microscopic phenomenon - it is a statistical phenomenon involving a huge number of cells. The result of the decay of a single radioactive atom can be thought of as a microscopic 'record' of a quantum event which can be preserved indefinitely. However, this record remains inaccessible until 'amplified' by an appropriate macroscopic instrument.

BRUCE GREGORY

Harvard-Smithsonian Center for

Astrophysics,

60 Garden Street, Cambridge,

Massachusetts 02138, USA

NATURE · VOL $352 \cdot 29$ AUGUST 1991
New possibility for planet

SIR - The entirely unexpected nature of the setting in which the new planet in orbit around pulsar PSR1829-10 was discovered $^{1}$ has resulted in much controversy about the sequence of events that led to the configuration now observed. It is widely presumed that the supernova explosion that gave birth to the neutron star was a type Ib or type II event, triggered by core collapse in a massive $\left(M \geqslant 8 M_{\odot}\right)$ progenitor. This assumption leads immediately to some serious problems, which have been considered by the planet's discoverers ${ }^{1}$ and also by Black in News and Views ${ }^{2}$.

Confronted by these difficulties, one might resort to any of a number of hypotheses. The planet may have formed after the supernova explosion or, perhaps, it was captured from a passing planetary system. Neither of these is attractive. In the first case, the pulsar is thought to be young, with a characteristic age estimated to be only about 1.25 Myr. This can scarcely be sufficient time to form a planet of mass $M \approx 10 M_{\oplus}$ in an environment which was, in all probability, thoroughly scoured by the supernova blast wave. It is not impossible that the planet was gravitationally captured from a system in transit through the pulsar's neighbourhood, but the required 'fine-tuning' of the encounter parameters makes such an event highly unlikely, especially given the relatively short interval during which it must have occurred. Bahcall $^{3}$ has raised the intriguing possibility that many, perhaps most, core collapses result in 'neutrino bombs', with very little in the way of an optical display; a nearby planet might survive such a catastrophe, although the short lifetime of the massive progenitor, and its immensity during the red supergiant phase remain causes for concern.

I suggest another possibility, that the supernova explosion which left the remnant PSR1829-10 was of type Ia, and that the progenitor was a single, massive hot white dwarf. An interesting model for such events has been described by Bowers and Deeming ${ }^{4}$, in which hot white dwarfs may exist at masses slightly above the Chandrasekhar limit for zero temperature electron-degenerate configurations because of pressure support from nondegenerate nuclei. For a white dwarf at temperature $T$, composed of material of mean atomic weight $A$, they argue that the critical mass $M_{\mathrm{Ch}}$ is given by

$$
M_{\mathrm{Ch}}(T) \approx M_{\mathrm{Ch}}(0)\left(1+2 \times 10^{-11} A T\right)
$$

This decreases as the star cools, and, when it falls below the actual mass, collapse occurs. What ensues is not clear, but Bowers and Deeming suggest mechanisms leading to an explosion which ejects the outer $0.1-0.2 M_{\odot}$ of the white dwarf, and leaves a neutron-star remnant. The appeal of this in the present context is clear. Because the star whose remnant is the white-dwarf presupernova need not be especially massive $\left(M \geqslant 1.5 M_{\odot}\right)$, it has a long lifetime, ample for the formation of a planetary system. Second, such stars do not expand to enormous radii as red giants (50-60 $R_{\odot}$ is typical) and, even if they do so as asymptotic giants, their low-mass envelopes are extremely tenuous. Thus, the orbital decay problem is eased, if not eliminated. Third, the mass of debris incident on a nearby planet after the supernova explosion is sharply reduced, thereby enhancing the planet's prospects for survival. Fourth, the central mass left after the outburst is about $80 \%$ of the precursor mass, so that the orbits of companion planets probably remain bound. Moreover, the model accounts naturally for the apparently complete absence of hydrogen features from supernova Ia spectra, which is not readily explained by models requiring mass transfer from the outer layers of a hydrogen-rich stellar companion, or by explosions in a common envelope ${ }^{5}$. At the same time, it obviates concerns about the stability of close planetary orbits in a binary system.

Peter C. Dawson

Department of Physics,

Trent University,

Peterborough, Ontario,

Canada K9J $7 B 8$

1. Bailes, M., Lyne, A. G. \& Shemar, S. L. Nature 353 26-28 (1991)

2. Black, D. Nature 352, 278 (1991).

3. Bahcall, J. N. Neutrino Astrophysics (Cambridge University Press, 1989. .

4. Bowers, R. \& Deeming, T. Astrophysics I Stars (Jones and Bartlett, Portola Valley, 1984).

5. Applegate, J. H. Astrophys. J. 370, 324-329 (1991)

\section{Ticking off}

SIR - It was with alarm that I read in News and Views that East Coast fever, which is caused by infection with the protozoan parasite Theileria parva, can also be transmitted by mosquitoes (J. Brady Nature 351, 695; 1991). It may be possible to "see tsetse flies from space", as the title of the article concerned has it, but it is clearly not possible to distinguish that ticks (C. P. Lounsbury, Transvaal Agric. J. 2, 4; 1903) rather than mosquitoes are the vectors of East Coast fever.

\section{Department of Parasitology}

DIRK DOBBELAERE

Institut for Animal Pathology,

University of Bern,

Bern CH3012,

Switzerland 